\begin{tabular}{|c|l|}
\hline Title & Site selective silicon adatom desorption using femtosecond laser pul se pairs and scanning tunneling microscopy \\
\hline Author(s) & Futaba, D. N.; Morita, R.; Y amashita, M.; Tomiy ama, S.; Shigekawa, H. \\
\hline Citation & $\begin{array}{l}\text { APPLIED PHY SICS LETTERS, 83(12), 2333-2335 } \\
\text { https://doi.org/L0.1063/1613361 }\end{array}$ \\
\hline Issue Date & 2003-09-22 \\
\hline Doc URL & http://hdl.handle.net/2115/5874 \\
\hline Rights & Copyright $\odot 2003$ A merican Institute of Physics \\
\hline Type & article \\
\hline File Information & APL83-12.pdf \\
\hline
\end{tabular}

Instructions for use 


\title{
Site-selective silicon adatom desorption using femtosecond laser pulse pairs and scanning tunneling microscopy
}

\author{
D. N. Futaba, ${ }^{\text {a) }}$ R. Morita, and M. Yamashita ${ }^{\text {b) }}$ \\ Department of Applied Physics, CREST, Japan Science and Technology Corporation (JST), \\ Hokkaido University, Sapporo 060-8628, Japan \\ S. Tomiyama \\ Department of Applied Physics, Hokkaido University, Sapporo 060-8628, Japan \\ H. Shigekawa \\ Institute of Applied Physics, 21st COE, CREST, Japan Science and Technology Corporation (JST), \\ University of Tsukuba, Tsukuba 305-8573, Japan
}

(Received 28 April 2003; accepted 28 July 2003)

\begin{abstract}
We performed an experimental study of silicon adatom desorption from the $\mathrm{Si}(111)-7 \times 7$ surface using femtosecond laser pulse pair excitation with $80 \mathrm{fs}$ pulse duration, $800 \mathrm{~nm}$ center wavelength, $300 \mathrm{~mW}$ average power, and a $100 \mathrm{MHz}$ repetition rate. Using scanning tunneling microscopy, we directly recorded the desorption characteristics at each delay setting for each of the four adatom binding sites. The study revealed a preferential dependence between the delay time and the adatom sites within a 66.6-1000 fs delay range. (C) 2003 American Institute of Physics.
\end{abstract}

[DOI: $10.1063 / 1.1613361]$

Bonding at surfaces holds fundamental importance in our understanding and manipulation of chemistry and materials. Among them, femtosecond (fs) laser-induced breaking or forming chemical bonds can provide crucial insight into these surface-related processes. A considerable amount of research has already been done on the study of the interaction between light and solid-state surfaces, ${ }^{1,2}$ in particular, on semiconducting surfaces, for both the fundamental understanding and the technological importance to solid state devices. Structural changes have been observed to occur as a result of this interaction using lasers, and electronic processes have been shown to play important roles when the laser intensity was below the melting threshold. ${ }^{3-5}$ Charge transfer and rearrangement are also thought to be key in making and breaking surface chemical bonds. In addition, the coupling of photoexcited electrons to the nuclear motion of the surface species and the time scale involved in bond dissociation and formation are two important issues. However, such optically induced dynamic surface phenomena have not been well studied at the atomic scale. ${ }^{6}$

The $\operatorname{Si}(111)-7 \times 7$ surface, whose structure consists of distortions from the ideal bonding tetrahedral $s p^{3}$ bonding geometry, relies heavily on electron-lattice interaction for structural stability. ${ }^{7}$ This reconstructed silicon surface presents four distinct adatom sites, which differ both electronically and dynamically ${ }^{8,9}$ and is thought they could serve as sites to excite localized vibrations. The purpose of this letter is to experimentally demonstrate that the fs delay-time difference in optical pulse pair excitation plays a role in the site-selective desorption of $\mathrm{Si}$ adatoms under below-melting power irradiation. Using a scanning tunneling microscope (STM) to directly observe the numbers of vacancies, we in-

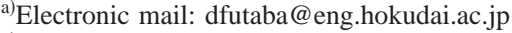

b) Author to whom correspondence should be addressed; electronic mail: mikio@eng.hokudai.ac.jp
}

vestigated the pulse pair delay-time dependence of $\mathrm{Si}$ adatom desorption from the $\mathrm{Si}(111)-7 \times 7$ surface. These findings can provide a selective fabrication technique for nanometer-scale devices as well as physical and chemical insight into ultrafast dynamic processes at surfaces with atomic scale resolution.

We performed a room temperature desorption study of $\mathrm{Si}$ adatoms from the $\mathrm{Si}(111)-7 \times 7$ surface using a fs laser-STM system [Fig. 1(a)]. The STM was a commercially purchased low-temperature ultrahigh vacuum instrument by UNISOKU Co., Ltd., Japan and was operated at a base pressure of $\sim 8.0 \times 10^{-11}$ Torr. Si(111) samples were cut from an $n$-type, As-doped $(\rho=0.001-0.005 \Omega / \mathrm{cm}) \mathrm{Si}(111)$ wafer and degassed under ultrahigh vacuum conditions at $\sim 600^{\circ} \mathrm{C}$ for 10 h. The samples were subsequently flash-heated at $\sim 1150^{\circ} \mathrm{C}$ for $10 \mathrm{~s}$ in order to form the $7 \times 7$ reconstruction. During flash-heating, the pressure never exceeded $1.5 \times 10^{-10}$ Torr.

Linearly polarized pulses from a mode-locked Ti:sapphire laser $(100 \mathrm{MHz}$ repetition rate, $80 \mathrm{fs}$ pulse width, 300 $\mathrm{mW}$ average power, and $800 \mathrm{~nm}$ central wavelength) were directed into a Michelson-type interferometric delay and were then focused to a $300 \mu \mathrm{m}$ diameter spot on the sample surface by a silver concave mirror (focal length of $300 \mathrm{~mm}$ ). The $p$-polarized (parallel to the reflection plane) laser pulses were incident to the surface at approximately $45^{\circ}$. During laser irradiation, the tip was withdrawn out of the path of the laser to avoid desorbing any adsorbed species that may have resided on the tip. The pulse pair delays ranged from 66.6 to $1000 \mathrm{fs}(15.0$ to $1.0 \mathrm{THz})$ with an irradiation time of $60 \mathrm{~min}$. The adatom removal yield was found roughly to be on the order of 5 vacancies per 20 unit cells.

After verifying sample cleanliness by STM and characterizing the number of pre-existing adatom vacancies by examining several thousand unit cells, we irradiated the surface. After allowing for thermal drift to settle, the surface was once again examined by STM. The desorption yield was determined by counting the number of vacancies for images 

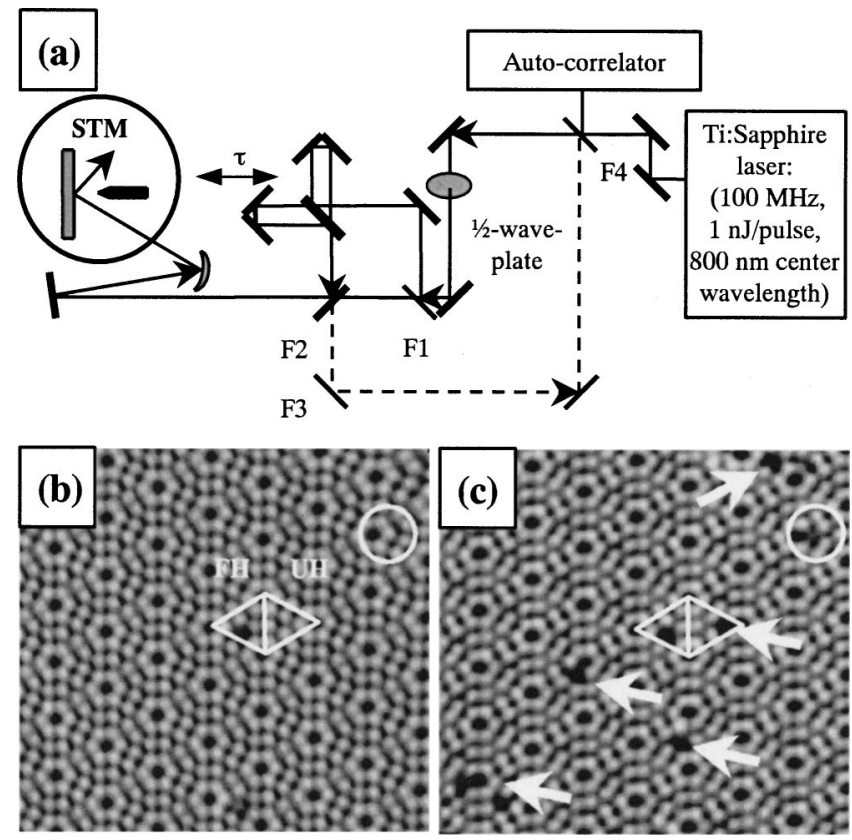

FIG. 1. (a) Pulses from a mode-locked Ti:sapphire laser (100 MHz repetition rate, 80 fs pulse width, $300 \mathrm{~mW}$ average power, and $800 \mathrm{~nm}$ central wavelength) are directed into the STM vacuum chamber after being diverted through Michelson-type interferometric delay. Flip mirrors (F1-F4) allow for pulse characterization during the experiment. (b) A STM image of the $7 \times 7$ surface before irradiation. $I=0.2 \mathrm{nA}$; bias $=1.5 \mathrm{~V}$. Both polarities were taken for each location to identify adsorbed gases. (c) A STM image after 1 $\mathrm{h}$ of irradiation of two pulses. $I=0.2 \mathrm{nA}$; bias $=1.5 \mathrm{~V}$. The circle indicates an adsorbed gas as identified from the negative bias image.

both before and after irradiation, and this procedure was repeated several times for each delay setting to confirm reproducibility. All STM observations were done using PtIr tips and were taken in constant-current mode $(0.20 \mathrm{nA}, 1.5 \mathrm{~V})$.

STM images of sampled regions before and after laser irradiation of $4 \mu \mathrm{J} / \mathrm{cm}^{2}$ fluence reveal that the number of vacancies increases after laser exposure [Figs. 1(b)-1(c)]. STM images were taken in both polarities in order to rule out the possibility that apparent vacancies were due to adsorbed gases, which can appear as vacancies in one polarity and not in the other. ${ }^{10,11}$ An example of such a case is marked with a circle in the image. Accordingly, by using this as a rule, we are confident that the dark spots are adatom vacancies.

Upon incrementally decreasing the delay (increasing the pulse pair frequency), we observed that the total desorption yield showed an increasing trend with the exception at 12 THz [Fig. 2(a)]. The profile of the total desorption yield suggested that significant desorption occurred only below $333 \mathrm{fs}$ (above $3 \mathrm{THz}$ ).

We then examined the images further and compared the center-to-corner adatom desorption ratios for each delay setting. We found that the ratio ranged in value from as low as 0.876 to as high as 22.7 for each delay setting [Fig. 2(b)]. This revealed a desorption preference which varied with the excitation delay time. In addition, it showed one delay setting $(8 \mathrm{THz})$ to have a particularly high selectivity for center sites. It seems that this result corresponds to the fact that the faulted-half $(\mathrm{FH})$-center adatoms have the most prominent peak at $8 \mathrm{THz}$ for the surface phonon spectrum (one of the vibrational frequencies of localized phonons at adatom sites), while the FH-corner adatoms have no peaks at this freDownloaded 08 Mar 2006 to 133.87 .26 .100 . Redistribution subject

\section{(a) Total Net Yield versus Excitation Frequency}

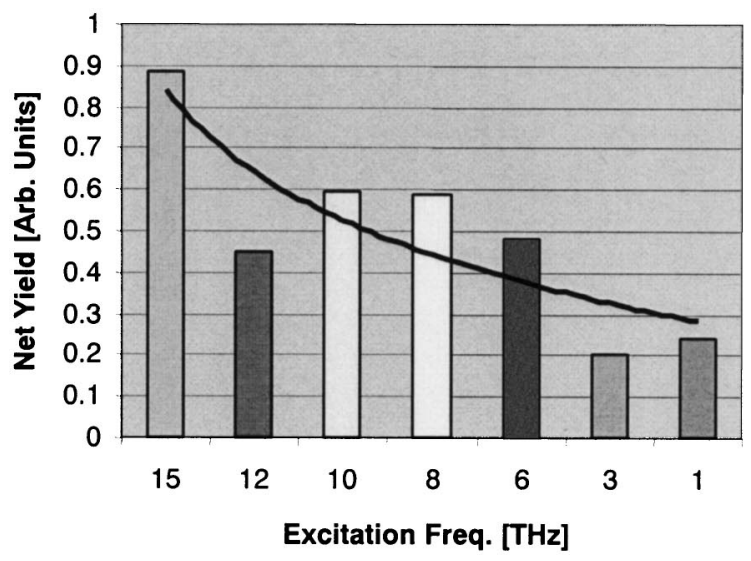

(b) Center/Corner Ration versus Freq.

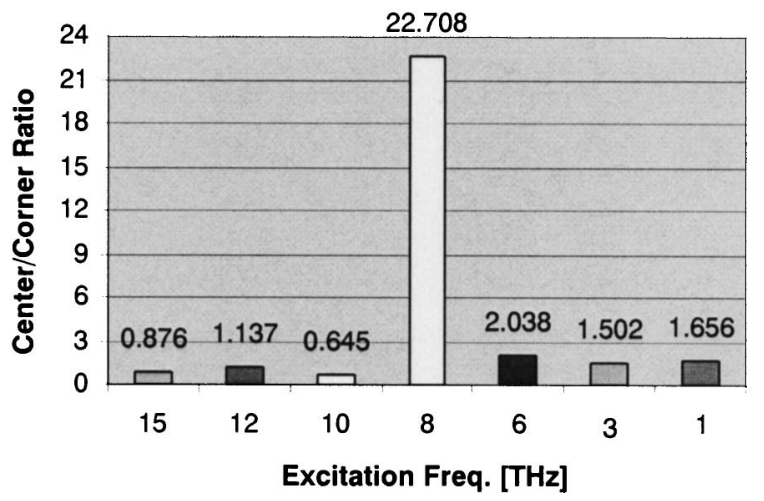

FIG. 2. (a) Total net yield as a function of excitation frequency. The trend indicates a general increase with higher frequencies (shorter delays). (b) The center to corner adatom ratio for each of the same excitation frequencies showing the preferential desorption between binding sites changes despite showing differences in total yield.

quency. ${ }^{9}$ This discovery prompted us to further investigate the adatom site dependence on the desorption yield.

As summarized in Fig. 3, a detailed investigation of the desorption yields for each binding site revealed subtle trends for each frequency setting and for each adatom site. This breakdown of data is presented with the binding sites ar-

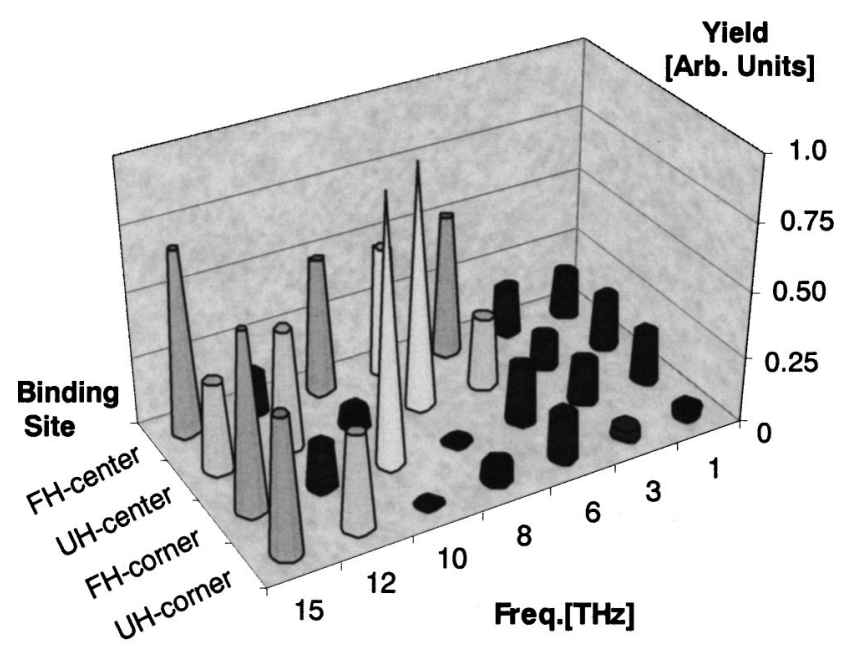

FIG. 3. Desorption yield as a function of binding site and excitation frequency with a pulse pair (1/delay time).

AlP license or copyright, see http://apl.aip.org/apl/copyright.jsp 
ranged in increasing binding energy site (i.e., FH-center, unfaulted-half (UH)-center, FH-corner, and UH-corner) ${ }^{12}$ to make any related trends more easily identifiable. The most obvious trends are the individual desorption profile for each binding site and the desorption profile at each excitation delay setting. Generally, FH-center sites showed the greatest level of desorption. This seems to correlate with the fact that the FH-center sites have the lowest binding energy among the four adatom sites. Throughout the excitation frequency spectrum, save the $1-3 \mathrm{THz}$ (to be discussed later) and the $12 \mathrm{THz}$ setting, the efficiency for the FH-center sites was roughly unvarying. The UH-center sites, however, showed a slightly varying behavior with being strongly efficient at 8 $\mathrm{THz}$ and considerably less efficient at 10,12 , and $15 \mathrm{THz}$. Similarly distinct peaks existed at 10 and $15 \mathrm{THz}$ for the FH-corner sites. The UH-corner sites exhibited maxima in the desorption yield at 12 and $15 \mathrm{THz}$.

Examining the profiles for each excitation setting, we observed that at lower frequencies, such as $1-3 \mathrm{THz}$, not only was there an insignificant level of desorption, there appeared to be no prominent site-preferred vacancies (Fig. 3). With higher frequencies, 6-12 THz, not only did the desorption efficiency increase, but also site-preferences emerged. At $6 \mathrm{THz}$, the desorption levels at all sites increased with a prominent peak for the FH-center sites. At $8 \mathrm{THz}$, highly selective profiles appeared with negligible desorption at two higher binding-energy sites (FH-corner and UH-corner) and distinct peaks at the two lower ones (FH-center and UHcenter). At 10 and $12 \mathrm{THz}$, in difference to prior trends where we observed preferences between center and corner sites, we observed a preference between subunits. At $10 \mathrm{THz}$, there was a marked preference to the FH-center and FH-corner sites desorption, while at $12 \mathrm{THz}$ this tendency was reversed. Finally, at $15 \mathrm{THz}$, the desorption at all sites showed increased yields while showing no clear preferential behavior.

We believe that the process governing desorption is a two-step excitation. The first pulse excites an electron in a surface bonding state to an intermediate state with a lifetime $\tau$, which is characteristic to the local electronic properties of the binding site. Additionally as deduced from Fig. 3, this lifetime is shorter than $333 \mathrm{fs}$. Using a single $1.55 \mathrm{eV}$ photon, with the intensity below the melting threshold, the probability for removing an adatom is virtually zero. However, if a second pulse interacts with the medium within the lifetime of the excited surface bonding state, its energy can add to the first. In such a case, local phonons around the adatom, whose electron contributes to the substrate bonding, are further excited, which gives rise to the possibility of bond breakage. This two-photon process must therefore depend on the delay time. Further, we believe that differences in the desorption preferences stem from differences in the local dynamic properties surrounding each adatom site. ${ }^{9}$ To be complete, detailed energy-band structure calculations are needed to locate the precise energy level involved.

More recent experimental results using $s$-polarized fs pulses showed insignificant levels of desorption for a variety of delay times. This result strongly suggests that surface heating associated with fs-laser irradiation is not the dominant desorption mechanism particularly at short delay times. Furthermore, it also suggests a dipole-like interaction between the adatom sites (including the dangling bonds) and the laser. Moreover, this gives rise to localized phonons modes oriented along the surface normal direction. ${ }^{9}$

In summary, the desorption characteristics of silicon adatoms from the $\mathrm{Si}(111)-7 \times 7$ surface were investigated using fs laser pulse pair excitation and direct observation with STM. A delay-time dependence on the overall desorption yield and desorption character was observed. With shortened delay time (increased excitation frequency), the total desorption yield increased. In addition, preferences among adatom binding sites were observed as a function of the delay time.

The authors gratefully acknowledge the helpful discussions with Professor C. Y. Fong.

${ }^{1}$ M. Vol Allmen, Laser-Beam Interaction with Materials (Springer, Berlin, 1987).

${ }^{2}$ Special issue on Laser-Induced Material Modifications, edited by P. J. Kelly, Opt. Eng. 28 (1989), entire issue.

${ }^{3}$ K. Ishikawa, J. Kanasaki, Y. Nakai, and N. Itoh, Surf. Sci. Lett. 349, L153 (1996).

${ }^{4}$ K. Ishikawa, J. Kanasaki, K. Tanimura, and Y. Nakai, Solid State Commun. 48, 913 (1996).

${ }^{5}$ J. Kanasaki, T. Ishida, K. Ishida, and K. Tanimura, Phys. Rev. Lett. 80, 4080 (1998)

${ }^{6}$ O. Takeuchi, R. Morita, M. Yamashita, and H. Shigekawa, Jpn. J. Appl. Phys., Part 1 41, 4994 (2002).

${ }^{7}$ K. Takayanagi, Y. Tanishiro, M. Takahashi, and S. Takahashi, J. Vac. Sci. Technol. A 3, 1502 (1985).

${ }^{8}$ W. Daum, H. Ibach, and J. E. Müller, Phys. Rev. Lett. 59, 1593 (1987).

${ }^{9}$ J. Kim, M.-L. Yeh, F. S. Khan, and J. W. Williams, Phys. Rev. B 52, 14709 (1995).

${ }^{10}$ I.-W. Lyo, P. Avouris, B. Schubert, and R. Hoffman, J. Phys. Chem. 94, 4400 (1990).

${ }^{11}$ J. J. Boland, Surf. Sci. 244, 1 (1991).

${ }^{12}$ Y. F. Zhao, H. W. Yang, J. N. Gao, Z. Q. Xue, and S. J. Pang, Surf. Sci. Lett. 406, L614 (1998). 